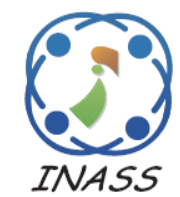

\title{
Adaptive Skin Colour Modelling for Hand and Face Segmentation
}

\author{
Shailendra Chandrakumar Badwaik ${ }^{1 *}$ \\ Shashikant Dnyandev Lokhande ${ }^{1}$ \\ ${ }^{1}$ Sinhgad College of Engineering, \\ Department of Electronics and Telecommunication Engineering, \\ Savitribai Phule Pune University, Pune, India \\ * Corresponding author's Email: shailendra.sppu@gmail.com
}

\begin{abstract}
A real-time skin colour segmentation is a prime concern in posture/hand gesture based sign language recognition. The developed novel method separate posture/hand gesture without any marker on any background by taking into account foreground skin colour. The proposed algorithm, segmenting dynamic posture/hand gesture done in front of the camera or from images or video database. The values of $\mathrm{YCgCr}$ are determined in real time and form $\mathrm{YCgCr}$ adaptive bound. These bound values are used to segment foreground skin colour from skin / non-skin colour background. The algorithm deployed on the embedded image processing hardware platform and any skin colour/tone it can adapt and used further to segment face/ hand gestures. The proposed adaptive skin colour algorithm accuracy for standard Ali Yawar Jung video dataset of ISL hand gestures is $92.64 \%$ and visually when two different signers performed same gestures the average accuracy is $91.82 \%$.
\end{abstract}

Keywords: Skin colour model, Hand gesture, $\mathrm{YCg} C r$, ASCM.

\section{Introduction}

Skin colour segmentation plays a prudent role in a broad range of image processing applications like gesture/posture detection and analysis, face detection and tracking, human-computer interface (HCI) system, content-based image retrieval (CBIR) systems, etc. Many researchers use skin colour segmentation approach as it involves less computation than the other segmentation methods.

Kakumanu, P., S. Makrogiannis, and N. Bourbakis in [1] explores the details of different skin colour in three different ways: first, different colour spaces used for skin modelling and detection. Second, different skin colour modelling and classification. Third, use skin-colour dynamic adaptation techniques[2, 3] for dynamically varying illumination and environmental conditions.

Skin colour modelling is done based on colour space like: Basic Colour Spaces [3] (RGB, normalised RGB, CIE-XYZ) [4], Perceptual colour spaces [5, 6] (HSI, HSV,
HSL, TSL), Orthogonal colour spaces [1] (YCbCr, YIQ, YUV, YES) perceptually uniform colour spaces [2, 3, 7, 8] (CIE-Lab and CIE-Luv) and Hybrid colour spaces [3, 9]. On the other hand skincolour classifications [5] done using Explicit skincolor space thresholding [10], Histogram model with Naïve Bayes classifier, Gaussian classifier : Single Gaussian models (SGM) [11], Gaussian mixture models (GMM) [12], Elliptical boundary model [13], Multi-layer perceptron (MLP) classifier [14], Selforganizing map (SOM) classifier [15], Maximum entropy classifier [16], Bayesian network (BN) classifier [7]. Each Skin colour modelling and classification method has its own merits and demerits. These methods play a prudent role in hand segmentation and recognition. In this paper $\mathrm{YCgCr}$ skin colour based adaptive skin segmentation model designed and used to identify Indian Sign Language (ISL) gestures in real time.

Hierarchy of Skin colour modelling is as shown in Fig. 1 as follows. The hierarchy of Skin colour classifiers classification is as shown in Fig. 2. 


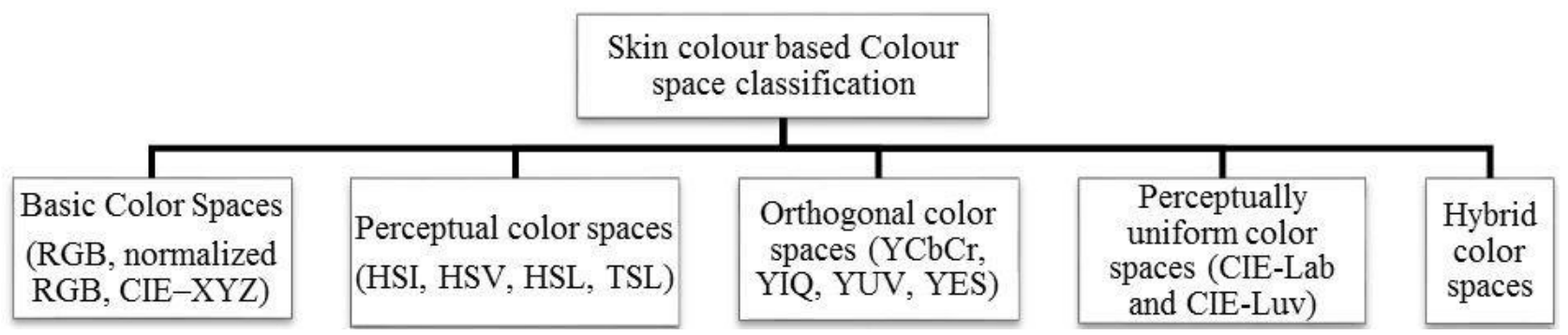

Figure. 1 Skin colour modelling based on colour space classification

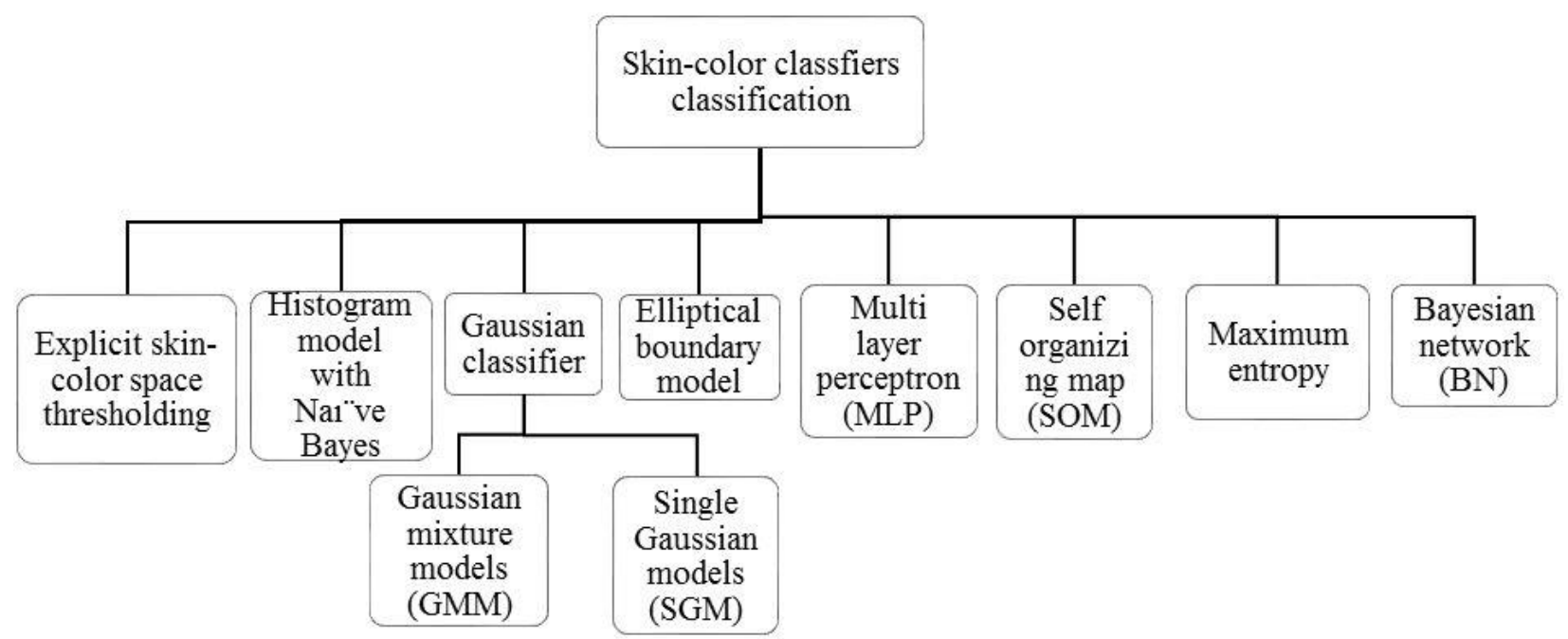

Figure. 2 Skin colour classifiers classification

The listed classifications are not limited to gesture recognition. Various researchers used sensors, clour gloves, markers, skin colour spaces and classification techniques for gesture recognition by creating fixed bounds or threshold values for $R G B, H S I, H S V, Y C g C r$ etc. These bounds or threshold values may work in specific conditions. Certainly, these fixed bounds or thresholds restricts real-time gesture recognition. This key issue addressed in proposed work. The main focus of this paper is for adaptive varying skin colour/tone modelling and detection for hand / face gesture(s) / posture(s) segmentation and detection. The proposed algorithm detects signer's variations in skin colours/ tones of Hand/ face gesture(s) / posture(s) which are done in front of camera for segmenting skin colour hand / face for gesture(s) / posture(s). The adaptive skin colour/tone model generates specific bounds for $\mathrm{Y}, \mathrm{Cg}$ and $\mathrm{Cr}$ in real time further these are used for segmenting skin colour Hand/ face gesture(s) / posture(s).

Researchers have suggested various schemes/methods for skin colour segmentation which works for specific bounded values of skin colour/tone. Problems of fixed $\mathrm{YCgCr}$ bound analysed as:

- Varying skin tone gives different bounds for skin segmentation
- In INDIA itself skin tone varies drastically for different states

- Unable to segment dark skin clour of African, Caucasian and Hispanic people

- Colour Histogram analysis indicate the presence of black colour in such skin tone

- Colour Histogram analysis generally gives skin tone as Red and Yellow colour

It indicates $\mathrm{YCgCr}$ model need to be adaptive an innovative technique is developed to detects signer's variations in skin colours/ tones of Hand/ face gesture(s) / posture(s) done in real time.

The present invention deals with recognition of hand gestured performed in front of the camera by separating signers skin colour region of interest mainly hand/face. Thus this technique generates adaptive skin colour/tone bounds of $Y, C g$ and $\mathrm{Cr}$ segments out hand/face from the human body for gesture/posture recognition. The algorithm is designed and deployed on image processing embedded platform.

This main contribution of this paper tackles several other features which are:

[1] For hand segmentation from posture proposed method does not require a sensor, marker, glove, etc. 
[2] Real-time recognising any signer's skin colour/tone.

[3] Separate palm from hand, face from the body in any background including skin colour/tone.

The core logic designed is in the form of functions tailored for image segmentation. Hence, the algorithm works for different backgrounds and varying signer's skin colour/tone. The proposed method will help hard in listening people to communicate seamlessly with normal human beings without using marker, sensor, glove, etc. kind of restrictions.

The proposed method gives better results regarding skin colour segmentation and we modified fixed thresholding $R G B$ to $\mathrm{YCgCr}$ conversation into adaptive skin colour model (ASCM) for efficient skin colour segmentation process. ASCM based three crops from signers forehand and backhand helped in identifying Indian Sign Language (ISL) hand gestures near to face of the signer, beneath the face of the signer, in front of the face of signer etc. Our proposed method also supports, visually selecting and recognising the single and both hand gestures and eliminate signers face. The first section introduces different skin colour model and overview of related work. The second section describes our Proposed Methodology for adaptive skin colour model. Experimental results with the suitable figures described in the third section for ISL hand gesture recognition using proposed ASCM skin colour segmentation method. This method gives a region of interest (ROI), i.e. palm from signers posture. These ROI objects used for features extraction; these further used for designing feature vector bounds and ISL hand gesture recognition. The fourth section gives conclusion and future work.

\section{Adaptive skin colour modelling (ASCM)}

Gesture recognition pertains to recognising different signer's skin colour/tone meaningful expressions of motion by a human, involving hands, arms, face, head and body. It is crucial in designing an intelligent and efficient human-computer interface. Gestures are used to convey the information and interact with the environment by any human being.

\subsection{Analysing skin colour by colour histogram}

Skin colour analysis is done using the colour histogram and shown in Figs. 1, 2, 3 and 4. The face of women shown in Fig. 3 is same except left side face is with makeup, and right side face in this figure is without makeup. The forehead skin sample showed besides the face. Colour histogram analysis of forehead skin sample used for building adaptive skin colour model. The colour histogram indicates makeup face with red, yellow and white colour bar chart, on the other hand, it differentiates without makeup face by indicating only red colour in a bar chart.

The skin colour across the globe is drastically varying which is analysed by the colour histogram. The faces indicated in Fig. 4 are African countries which are dark in colour. The same analysis is carried out by colour histogram for left side woman face by indicating red and black bar chart. In right side face of man indicates red and black bar chart but its level is different than left side woman face in Fig. 4. The presence of black colour indicates it is dark skin colour. For the dark skin colour, the colour histogram shows the intensity of black bar. The earlier face in Fig. 3 is white skin woman face; colour histogram indicates the absence of a black bar. The light intensity plays a vital role in deciding black colour presence. The white skin faces shown in Fig. 5 is captured by keeping light source on the left side of the face; hence half of the face on the left side is brighter than right half of face. The shadow of nose reflects the right side of the face which in turn makes right half of face dark in colour. The colour histogram analysis for forehead skin sample of white skin man shown in Fig. 5 indicates presence of black bar along with red colour. In this case, light distributed unevenly on the face.

Fig. 6 indicates white skin woman face. Colour histogram analysis for the left side white woman forehead skin sample shows the presence of red colour by a bar chart.

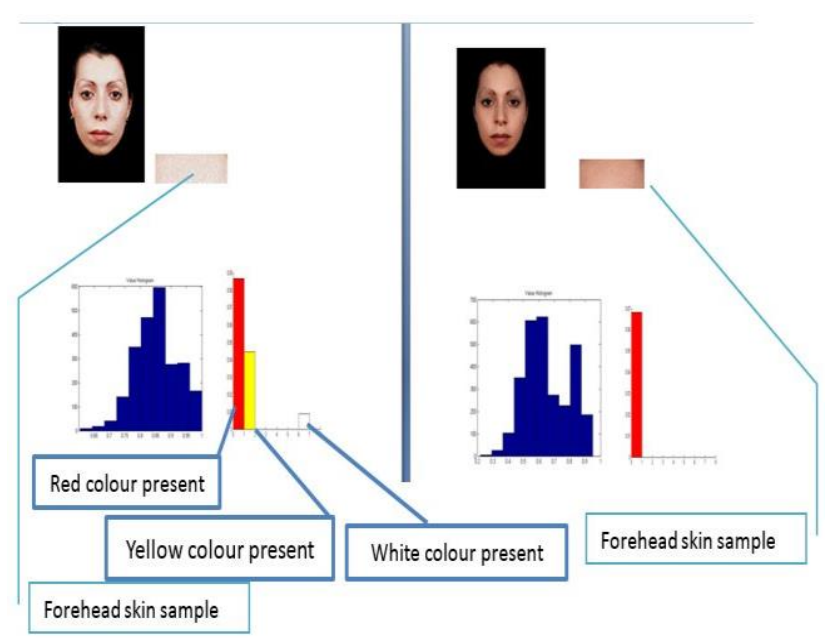

Figure. 3 Colour histogram analysis of skin colour tone with the presence of red and yellow colour for the same signer in different light conditions 


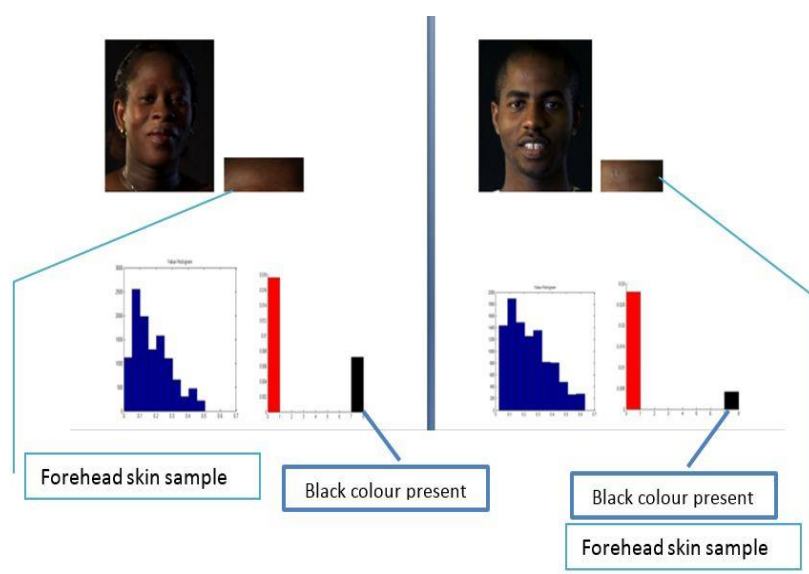

Figure. 4 Colour histogram analysis of skin colour tone with the presence of red and black colour.
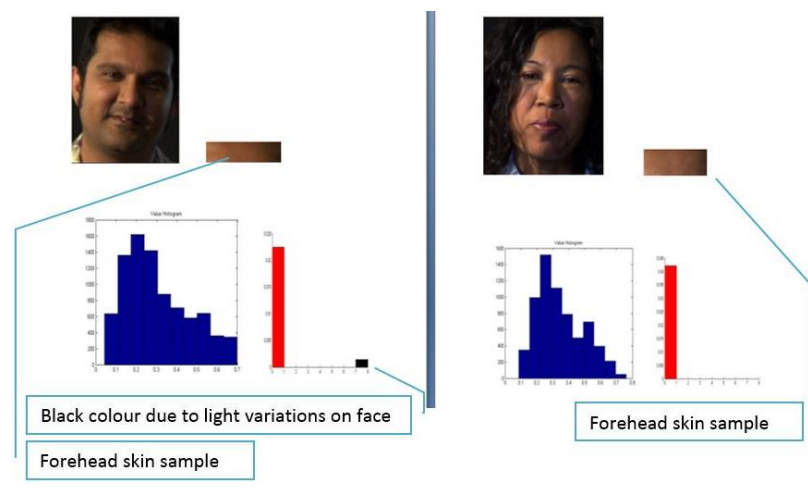

Figure. 5 Colour histogram analysis of skin colour tone with the presence of red and black colour.
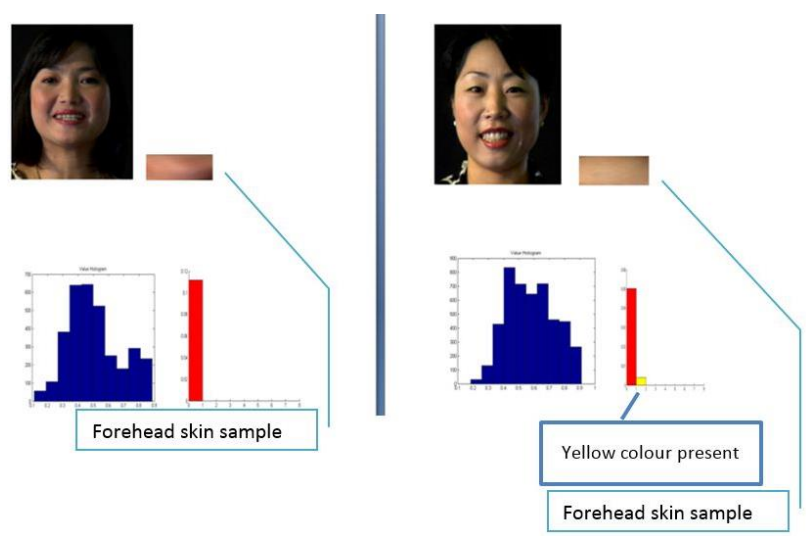

Figure. 6 Colour histogram analysis of skin colour tone with the presence of red and yellow colour.

The colour histogram analysis for right-hand side white women forehead skin sample shows the presence of red and yellow colour by a bar chart. The persons having white skin colour across the world is varying, its analysis made by the colour histogram.

\subsection{Analysing $Y C g C r$ Values of Skin Colour}

The experiments are carried out for two different signers. The 13 megapixel HD camera is used to capture the signer's face and hand image. These images are processed to find $\mathrm{YcgCr}$ values for facial, skin and hand. Equations for $R G B$ to $Y C g C r$ conversion mentioned in [17] are used to compute $\mathrm{YcgCr}$ values. At three different places like the forehead, face and nose the face image is randomly cropped. The user can take any three crops of skin. Tables 1, 2 and 3 have five rows and ten columns. The first crop yields the values of a second row. Similarly, second and third crop gives values of the third and fourth row respectively. The most minimum and most maximum value of $\mathrm{Y}, \mathrm{Cg}$ and $\mathrm{Cr}$ are computed. The last row of Table 1 shows three crops, the most minimum value $Y_{\min }$ is 18.535 , and the most maximum, $Y_{\max }$ is 151.494 . Similarly $C g_{\text {min }}$, $C g_{\max }$ and $C r_{\text {min }}, C r_{\text {max }}$ values determined in Tables 1,2, and 3. The average values $\mathrm{Y}, \mathrm{Cr}$ and $\mathrm{Cr}$ are computed using the minimum and maximum values of $\mathrm{Y}, \mathrm{Cr}$ and $\mathrm{Cr}$. In Table $1 Y_{\text {avg }}$ is 85.014 determined by taking an average of $Y_{\min }$ and $Y_{\max }$ value. Similarly, $C g_{\min }, C g_{\max }, C r_{\min }$, and $C r_{\text {max }}$ values are used to determine $C g_{\text {avg }}$ and $\mathrm{Cr}$ avg.

\subsection{Designing an adaptive skin colour model}

For taking three crops and find $\mathrm{Y}, \mathrm{Cg}$ and $\mathrm{Cr}$ value the frame number 14 considered. This frame should consist skin colour part. The earlier frames skipped as signer gets enough time to bring bare hand in front of the camera. The $R G B$ image captured by camera need light compensation. It is done using Eqs. (1) to (4). For taking three crops and find $Y, C g$ and $\mathrm{Cr}$ value the frame number 14 considered. This frame

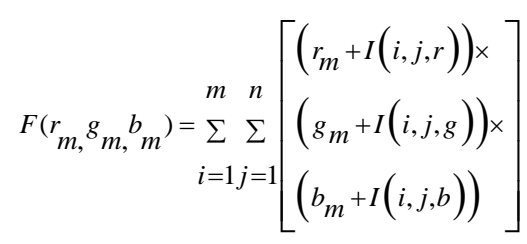

Where, the frame $F\left(r_{m}, g_{m}, b_{m}\right)$, is the outcome of input image components of red, green and blue colour planes as $I(i, j, r), I(i, j, g)$ and $I(i, j, b)$.

$$
r_{(i, j)}=\frac{r_{m}}{m \times n} ; g_{(i, j)}=\frac{g_{m}}{m \times n} ; b_{(i, j)}=\frac{b_{m}}{m \times n}
$$

The average of $R G B$ objects are computed like red as $r(i, j)$, green as $g(i, j)$ and blue as $b(i, j)$.

$$
k=\frac{\eta(i, j)+g(i, j)+b(i, j)}{3}
$$


Table 1. Variations in $\mathrm{YCgCr}$ values for skin colour/tone of Libyan Signer's Face

\begin{tabular}{|c|c|c|c|c|c|c|c|c|}
\hline$Y_{\min }$ & $Y_{\max }$ & $C g_{\min }$ & $C g_{\max }$ & $\mathrm{Cr}_{\text {min }}$ & $\mathrm{Cr}_{\text {max }}$ & $Y_{\text {avg }}$ & $C g_{\text {avg }}$ & Cravg \\
\hline 41.087 & 104.639 & 121.473 & 126.197 & 125.386 & 137.520 & -- & -- & -- \\
\hline 18.535 & 151.494 & 119.611 & 126.849 & 128.664 & 145.722 & -- & -- & -- \\
\hline 34.831 & 85.509 & 119.717 & 126.545 & 127.143 & 138.777 & -- & -- & -- \\
\hline 18.535 & 151.494 & 119.611 & 126.849 & 125.386 & 145.722 & 85.014 & 123.230 & 135.554 \\
\hline
\end{tabular}

Table 2. Variations in $\mathrm{YCgCr}$ values for skin colour/tone of Cameroon Signer's Face

\begin{tabular}{rllllllllc}
\hline & $Y_{\min }$ & $Y_{\max }$ & $C g_{\min }$ & $C g_{\max }$ & $C r_{\min }$ & $C r_{\max }$ & $Y_{\text {avg }}$ & $C g_{\text {avg }}$ & $C r_{\text {avg }}$ \\
\hline 50.326 & 111.208 & 119.581 & 123.443 & 132.310 & 141.830 & -- & -- & -- \\
& 19.595 & 137.990 & 115.342 & 124.594 & 131.657 & 151.850 & -- & -- & -- \\
33.973 & 82.074 & 116.100 & 123.836 & 133.117 & 147.529 & -- & -- & -- \\
19.595 & 137.990 & 115.342 & 124.594 & 131.657 & 151.850 & 78.792 & 119.968 & 141.753 \\
\hline
\end{tabular}

Table 3. Variations in $\mathrm{YCgCr}$ values for skin colour/tone of Libyan Signer's Hand

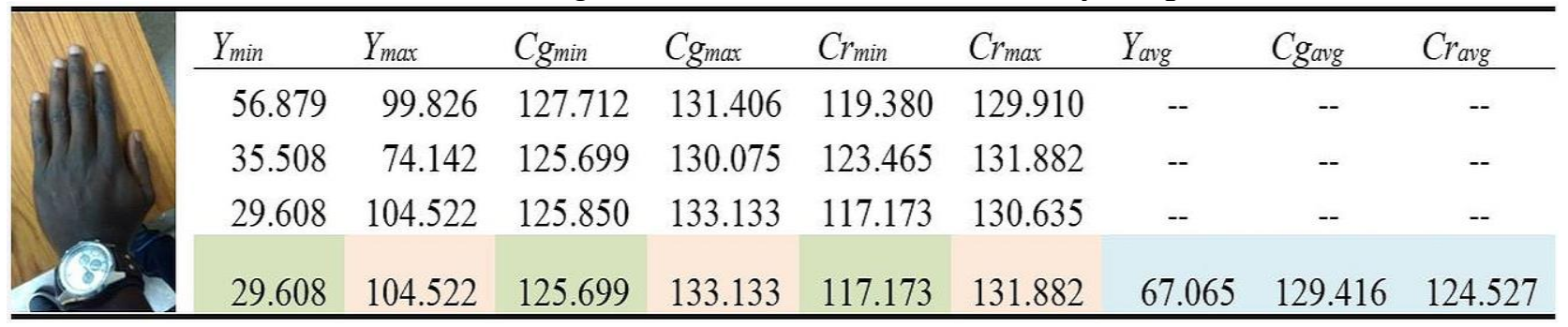

The average of all three planes red, green and blue is computed using Eq. (3).

$$
L(i, j)=\sum_{i=1}^{m} \sum_{j=1}^{n}\left[\begin{array}{l}
\left(\frac{\operatorname{frame}(r) \cdot k}{r}\right) \times\left(\frac{\operatorname{frame}(g) \cdot k}{g}\right) \times \\
\left(\begin{array}{l}
\text { frame }(b) \cdot j) \\
b_{(i, j)}
\end{array}\right)
\end{array}\right]
$$

Where $\mathscr{L}(i, j)$ is a light compensated image.

The transformation of $R G B$ to $Y C g C r$ done as discussed in[17]. To make system adaptive, $\mathrm{YCgCr}$ to binary conversion is done using Eq. (5) is designed as follows

$$
\sum_{\mathrm{i}=1 \mathrm{j}=1}^{\mathrm{m}} \sum^{\mathrm{n}} \mathrm{P}(\mathrm{i}, \mathrm{j})=\left\{\begin{array}{l}
1 ; \mathrm{II}(\mathrm{i}, \mathrm{j}, 1) \geq Y_{\text {avg }} \& C g_{\text {min }} \geq \mathrm{I}(\mathrm{i}, \mathrm{j}, 2) \leq \\
\left.C g_{\text {max }} \& C r_{\text {min }} \geq \mathrm{I}(\mathrm{i}, \mathrm{j}, 3) \leq C r_{\text {max }}\right] \\
0 ; \text { otherwise }
\end{array}\right.
$$

Where $Y_{\text {avg }}, C g_{\min }, C g_{\max }, C r_{\min }$ and $C r_{\max }$ are computed as follows

$$
\begin{gathered}
Y_{\text {min }}=\min _{1 \leq k \leq 3} \sum_{i=1}^{m} \sum_{j=1}^{n} C_{k}(i, j) \\
Y_{\text {max }}=\max _{1 \leq k \leq 3} \sum_{i=1}^{m} \sum_{j=1}^{n} C_{k}(i, j)
\end{gathered}
$$




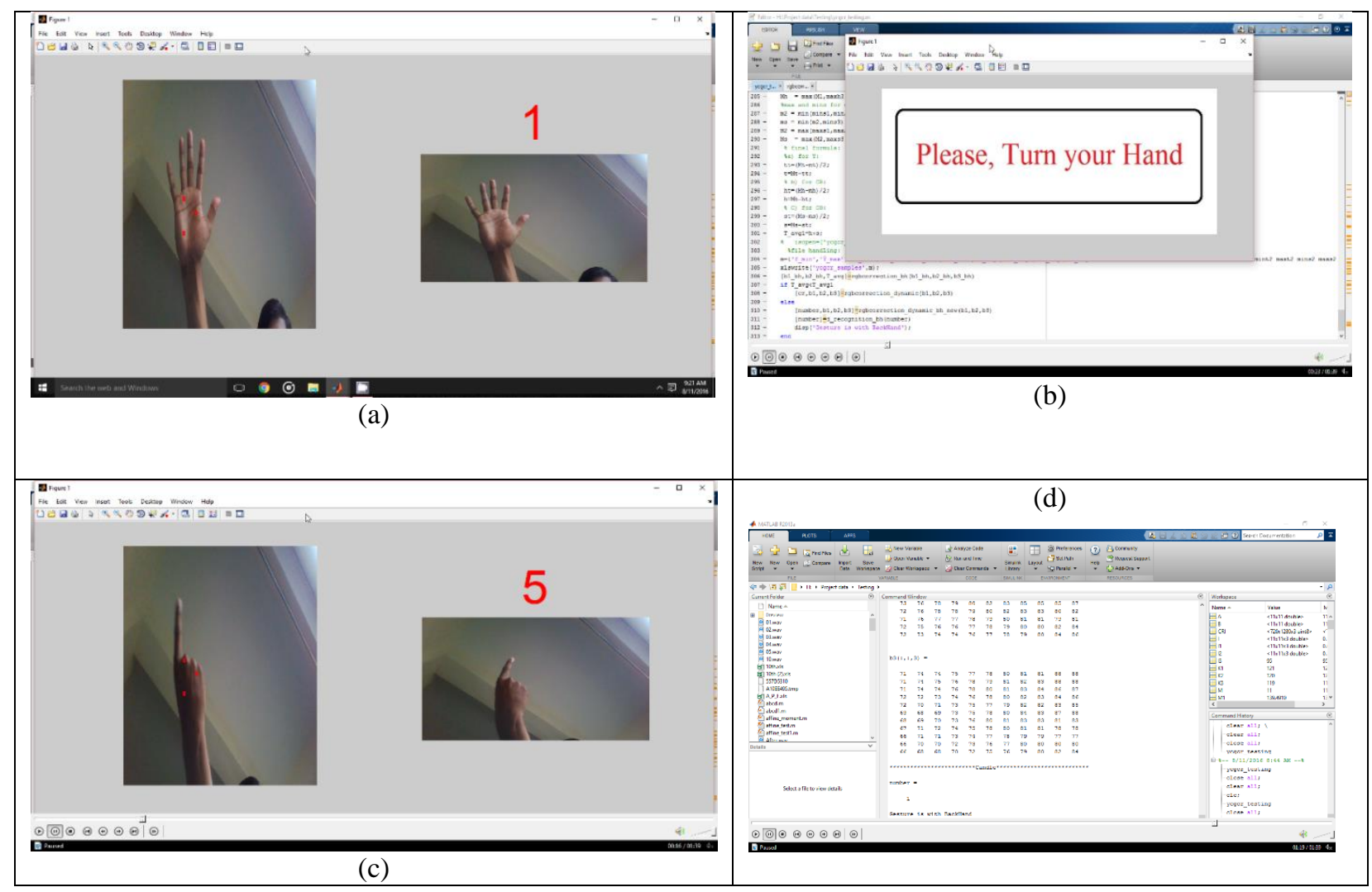

Figure. 7 Skin colour modelling: (a) ASCM for forehand skin colour processing, (b) Indication to user, (c) ASCM for backhand skin colour processing, and (d) Crop samples for ISL Recognized hand gesture 'Candle'

Table 4. Forehand and backhand $Y C g C r$ values for ISL gesture 'one and 'candle'

\begin{tabular}{|c|c|c|c|c|c|c|c|c|c|c|}
\hline \multicolumn{10}{|c|}{ Forehand $Y C g C r$ Values } \\
\hline$Y_{\min }$ & $Y_{\max }$ & $C g_{\min }$ & $C g_{\max }$ & $C r_{\min }$ & $C r_{\max }$ & $Y_{\text {avg }}$ & $C g_{\text {avg }}$ & $C r_{\text {avg }}$ & $T_{\text {avg }}$ \\
\hline 115.53 & 142.31 & 123.55 & 127.82 & 130.40 & 135.03 & 0.00 & 0.00 & 0.00 & 0.00 \\
\hline 138.13 & 151.78 & 125.06 & 128.92 & 127.92 & 132.54 & 0.00 & 0.00 & 0.00 & 0.00 \\
\hline 127.55 & 143.71 & 122.28 & 124.99 & 132.97 & 137.52 & 133.66 & 125.60 & 132.72 & 258.32 \\
\hline \multicolumn{10}{|c|}{ Backhand $Y C g C r$ Values } \\
\hline$Y_{\min }$ & $Y_{\max }$ & $C g_{\min }$ & $C g_{\max }$ & $C r_{\min }$ & $C r_{\max }$ & $Y_{\text {avg }}$ & $C g_{\text {avg }}$ & $C r_{\text {avg }}$ & $T_{\text {avg }}$ \\
\hline 107.15 & 138.83 & 126.74 & 130.50 & 125.99 & 131.79 & 0.00 & 0.00 & 0.00 & 0.00 \\
\hline 135.57 & 156.18 & 129.76 & 129.11 & 130.38 & 131.79 & 0.00 & 0.00 & 0.00 & 0.00 \\
\hline 134.33 & 154.46 & 126.17 & 128.94 & 128.62 & 135.07 & 131.66 & 128.33 & 130.53 & 258.86 \\
\hline
\end{tabular}

$$
C r_{\max }=\max _{1 \leq k \leq 3} \sum_{i=1}^{m} \sum_{j=1}^{n} C_{k}(i, j)
$$

The Algorithm for Adaptive skin colour/tone determination

Step1 From face/hand RGB image take three different skin colour crop of as $C_{k}(i, j)$ for $k=1,2,3$.

Step 2 Sort row wise and column wise pixels of each crop using Eqs. (6) to (11) to obtain values of $Y_{\text {avg }}, C g_{\min }, C g_{\max }, C r_{\min }$ and $C r_{\max }$
Step 3 Find Most Minimum and the Most Maximum Pixel value for $Y$ from Step 2

Step 4 Compute: $Y_{\text {avg }}=\left(Y_{\max }+Y_{\min }\right) / 2$

Step 5 Find Most Minimum and Most Maximum Pixel Value of $C g$ from Step 2

Step 6 Compute: $C g_{\text {avg }}=\left(C g_{\max }+C g_{\min }\right) / 2$

Step 7 Find Most Minimum and Most Maximum Pixel Value of Cr from Step 2 
Step 8 Compute: $C r_{\text {avg }}=\left(C r_{\max }+C r_{\min }\right) / 2$

Step 9 Set bound as

$$
\begin{aligned}
& {\left[I(i, j, 1)>Y_{\text {avg }} \& C g_{\text {min }}>I(i, j, 2)<C g_{\max } \&\right.} \\
& \left.C r_{\text {min }}>I(i, j, 3)<C r_{\text {max }}\right]
\end{aligned}
$$

\section{Results and discussion}

The binary segmented hand gestures from a posture of signer are segmented using Eq. (5). The above algorithm is used to segment hand gestures from standard Indian Sign Language (ISL) video database. The results of ten different ISL tabulated in Table 4. The database selected to segment gesture made by single hand, both hand, hand moving toward the face, hand moving away from the face, hand aside from the face, both hand overlap / mixed, etc. The proposed algorithm based the novel mathematical model improves the signers palm and face segmentation results compared to the work mentioned in [18, 19]. Above mathematical model designs the ASCM and based on that each three crops extracts total $11 \mathrm{X} 11$ matrix elements of the complete input image to get signers skin colour components. The design is as shown in Fig. 7 (a) where three red colour squares indicate the crop position to hold skin colour. In this signer need to hold his forehand/backhand such a way that skin colour covers these three squares. The system designed in such a manner that user gets enough time to place his/her hand on 3 cropping squares. A 10 down to 0 counter is imitated on the screen to overlap hand/skin portion in front of 3 squares. The image009.jpg, i.e. $9^{\text {th }}$ frame used for forehand processing. Further user gets a message on the screen to turn his/hand; the system is going to extract skin components of backhand. It illustrated in Fig. 7 (b). This time 5 down to 0 counts imitated, and the signer is supposed to overlap his backhand skin colour. The frame image_bh004.jpg, i.e. frame 14th (frames elapsed in case of forehand are 10 plus the next frames elapsed in the case for backhand are 4) used for backhand skin colour extraction. The Fig. 7 (c) illustrate it. ASCM based forehand and backhand $\mathrm{YCgCr}$ values indicated in Table 4. The segmented binary hand gestures as shown in the last column of Table 5 are further treated to extract different features. The prominent features utilised for gesture recognition are an area, perimeter, Euclidian distance, centroid, etc. Three different signers database is used to extract feature values bounds. Ali Yawar Jung designs standard ISL database. Indian government frames this organisation and the database accepted across India from the year 2017.

We replicated the some ISL database using two more signers, and that database is also used to generalise feature values and bounds. The min and max value is used to create the feature bound. Feature vectors and universal bounds used for real-time hand gesture recognition. This feature vector values indicated in Table 6. The notations used in Table 6 are AYG: Ali Yawar Jung (The Indian Organization Developing Standard ISL Database), FS: First Signer, SS: Second Signer, UB: Universal Bound. Column second and third specifies extracted feature values of minimum and maximum area dented as $\min _{-} A$ and $\max A$. Similarly, four and fifth represents extracted feature values of minimum, and maximum perimeter denoted in the table as $\min _{-} P$ and $\max _{-} P$, Column sixth and seventh specify extracted feature values of minimum, and maximum Euclidian distance dented as $\min _{-} E D$ and $\max _{-} E D$. Column eight, nine, ten and eleven represents extracted feature values of minimum and maximum centroid as $\min _{-} C 1$, $\min \_C 2, \max _{-} C 1$ and, $\max _{-} C 2$.

Fig. 8 shows the different steps involved in real time hand segmentation by proposed adaptive skin colour model. The hand segmented from posture by blob analysis, and skin colour face blob omitted over skin colour hand blob by area feature. Further Fig. 8 shows the Recognition of ISL gesture four in real time using same adaptive skin colour model and feature extraction by segmenting open fingers.

Tables 7 and 8 indicate confusion matrix when signer 1 and signer 2 make gestures in front of the camera.

The average accuracy for two signers is $91.82 \%$. The algorithms track skin colour of hands as shown in Figs. 8 and 9. The bounds designed using Table 6 are deployed in the algorithm to recognise the ISL hand gestures. Table 8 indicates the confusion matrix of video dataset gesture recognition. On the contrary, gestures are shown in Table 9 with same bounds of Table 6 give an accuracy of $92.64 \%$ for the standard 10 ISL. 
Table 5. Segmented binary hand gesture from posture

\begin{tabular}{|c|c|c|c|c|}
\hline $\begin{array}{l}\text { Sr. } \\
\text { No. }\end{array}$ & $\begin{array}{c}\text { ISL } \\
\text { Gesture } \\
\text { Name } \\
\end{array}$ & Database Analysis & ISL Gesture & $\begin{array}{l}\text { Segmented Binary } \\
\text { Hand Gesture }\end{array}$ \\
\hline 01 & Tenth & $\begin{array}{l}\text { HGR with both hands with all ten } \\
\text { fingers open }\end{array}$ & & \\
\hline 02 & Little & $\begin{array}{l}\text { HGR with a single hand and two } \\
\text { fingers open }\end{array}$ & & \\
\hline 03 & A Lot & $\begin{array}{l}\text { HGR with both hands moving } \\
\text { aside the face and all open fingers }\end{array}$ & & \\
\hline 04 & Enter & $\begin{array}{l}\text { HGR with overlap both hands and } \\
\text { lower hand moving front and back }\end{array}$ & & \\
\hline 05 & Late & $\begin{array}{l}\text { HGR with mixing both hand and } \\
\text { upper hand moving up and down }\end{array}$ & & \\
\hline 06 & Pass & $\begin{array}{l}\text { HGR with both hand and open } \\
\text { thumb }\end{array}$ & & \\
\hline 07 & Quite & $\begin{array}{l}\text { HGR with a hand in front of the } \\
\text { face }\end{array}$ & & \\
\hline 08 & Search & $\begin{array}{l}\text { HGR with left hand aside face } \\
\text { with an open little finger }\end{array}$ & & \\
\hline 09 & Wait & $\begin{array}{l}\text { HGR with a single hand with an } \\
\text { open palm }\end{array}$ & & \\
\hline 10 & Fail & $\begin{array}{l}\text { HGR with a single hand with } \\
\text { thumb moving downward }\end{array}$ & & \\
\hline
\end{tabular}


Table 6. Feature Vectors and Bounds for ISL Hand Gestures

\begin{tabular}{|c|c|c|c|c|c|c|c|c|c|c|}
\hline \multirow{2}{*}{$\mathrm{Col} \rightarrow$} & \multicolumn{2}{|c|}{ Area } & \multicolumn{2}{|c|}{ Perimeter } & \multicolumn{2}{|c|}{ Euclidian Distance } & \multicolumn{4}{|c|}{ Centroid } \\
\hline & 1 & 2 & 3 & 4 & 5 & 6 & 7 & 8 & 9 & 10 \\
\hline Tenth & min_A & $m a x \_A$ & min_P & max_P & $m i n \_E D$ & max_ED & $\mathrm{min}_{-} \mathrm{Cl}$ & max_Cl & min_C2 & $\max _{-} C 2$ \\
\hline AYG & 3711.00 & 5346.00 & 451.61 & 584.76 & 97.07 & 116.66 & 297.23 & 301.75 & 341.71 & 439.44 \\
\hline FS & 2501.00 & 3099.00 & 427.91 & 531.95 & 79.33 & 88.82 & 217.04 & 290.78 & 265.48 & 306.39 \\
\hline SS & 1787.00 & 2724.00 & 246.65 & 421.28 & 67.45 & 83.28 & 213.51 & 291.66 & 289.30 & 422.95 \\
\hline UB & 1787.00 & 5346.00 & 246.65 & 584.76 & 67.45 & 116.66 & 213.51 & 301.75 & 265.48 & 439.44 \\
\hline ALittle & $\min \_A$ & max_A & $\min \_P$ & max_P & $m i n \_E D$ & max_ED & min_Cl & max_Cl & min_C2 & max_C2 \\
\hline AYG & 1253.00 & 1332.00 & 205.58 & 216.55 & 39.94 & 41.18 & 104.79 & 106.05 & 172.07 & 179.80 \\
\hline FS & 829.00 & 908.00 & 172.27 & 190.17 & 32.49 & 34.00 & 110.50 & 111.52 & 189.78 & 194.74 \\
\hline SS & 801.00 & 906.00 & 141.20 & 180.17 & 31.94 & 33.96 & 100.15 & 118.32 & 168.38 & 218.58 \\
\hline UB & 801.00 & 1332.00 & 141.20 & 216.55 & 31.94 & 41.18 & 100.15 & 118.32 & 168.38 & 218.58 \\
\hline A Lot & min_A & max_A & $\min \_P$ & max_P & min_ED & max_ED & min_Cl & max_Cl & min_C2 & max_C2 \\
\hline AYG & 3289.00 & 3492.00 & 386.05 & 407.02 & 91.49 & 94.27 & 292.77 & 295.88 & 297.16 & 308.50 \\
\hline FS & 5437.00 & 5966.00 & 546.53 & 560.29 & 117.65 & 123.26 & 248.31 & 382.22 & 258.54 & 492.67 \\
\hline SS & 2455.00 & 2663.00 & 366.59 & 408.89 & 79.06 & 82.30 & 311.22 & 316.08 & 443.95 & 497.47 \\
\hline UB & 2455.00 & 5966.00 & 366.59 & 560.29 & 79.06 & 123.26 & 248.31 & 382.22 & 258.54 & 497.47 \\
\hline Enter & min_A & max_A & min_P $P$ & max_P & min_ED & max_ED & min_Cl & max_Cl & min_C2 & max_C2 \\
\hline AYG & 1378.00 & 1495.00 & 167.68 & 184.07 & 41.89 & 43.63 & 140.42 & 142.62 & 202.98 & 220.84 \\
\hline FS & 904.00 & 1270.00 & 144.27 & 231.28 & 33.93 & 55.90 & 127.26 & 55.03 & 176.79 & 389.53 \\
\hline SS & 1298.00 & 1673.00 & 201.68 & 232.79 & 56.71 & 65.15 & 255.23 & 266.31 & 207.81 & 496.30 \\
\hline UB & 904.00 & 1673.00 & 144.27 & 232.79 & 33.93 & 65.15 & 127.26 & 266.31 & 176.79 & 496.30 \\
\hline Late & min_A & max_A & min_P & max_P & min_ED & max_ED & min_Cl & max_Cl & min_C2 & max_C2 \\
\hline AYG & 996.00 & 1901.00 & 144.51 & 226.17 & 35.61 & 49.20 & 142.61 & 150.15 & 202.45 & 221.32 \\
\hline FS & 1258.00 & 1441.00 & 225.97 & 276.94 & 56.36 & 0.33 & 259.89 & 265.45 & 486.25 & 507.24 \\
\hline SS & 1880.00 & 1977.00 & 212.75 & 259.28 & 48.93 & 50.17 & 173.20 & 178.19 & 284.83 & 287.83 \\
\hline UB & 996.00 & 1977.00 & 144.51 & 276.94 & 35.61 & 60.33 & 142.61 & 265.45 & 202.45 & 507.24 \\
\hline Pass & min_A & max_A & min_P & max_P & min_ED & max_ED & $\min \_C 1$ & max_C1 & min_C2 & $\max \_C 2$ \\
\hline AYG & 2292.00 & 2474.00 & 315.18 & 333.97 & 76.38 & 79.36 & 288.68 & 293.08 & 306.10 & 377.86 \\
\hline FS & 222.00 & 587.00 & 79.36 & 192.91 & 23.76 & 38.66 & 261.80 & 68.49 & 406.21 & 460.72 \\
\hline SS & 15 & 1627.00 & 225.34 & 248.75 & 62.23 & 35 & 34 & 9.87 & 394.82 & 471.41 \\
\hline UB & 222.00 & 2474.00 & 79.36 & 333.97 & 23.76 & 79.36 & 252.34 & 293.08 & 306.10 & 471.41 \\
\hline Quite & min_A & max_A & min_P & max_P & min_ED & max_ED & min_Cl & max_C1 & min_C2 & max_C2 \\
\hline AYG & 3177.00 & 3703.00 & 272.45 & 357.36 & 63.60 & 68.66 & 142.06 & 145.20 & 108.29 & 130.02 \\
\hline FS & 1330.00 & 1579.00 & 236.65 & 287.62 & 41.15 & 44.84 & 132.55 & 134.90 & 82.22 & 88.98 \\
\hline SS & 1978.00 & 2115.00 & 310.69 & 356.39 & 50.18 & 71.41 & 125.92 & 249.79 & 109.16 & 278.49 \\
\hline UB & 1330.00 & 3703.00 & 236.65 & 357.36 & 41.15 & 71.41 & 125.92 & 249.79 & 82.22 & 278.49 \\
\hline Search & & max_A & min_P & max_P & min_ED & max_ED & min_Cl & max_Cl & min_C2 & max_C2 \\
\hline AYG & 1107.00 & 1404.00 & 138.23 & 172.95 & 37.54 & 42.28 & 91.03 & 141.51 & 134.28 & 202.67 \\
\hline FS & 406.00 & 710.00 & 78.53 & 150.91 & 22.74 & 30.07 & 81.60 & 121.57 & 169.69 & 232.31 \\
\hline SS & 562.00 & 840.00 & 122.43 & 144.67 & 26.75 & 32.70 & 95.49 & 142.72 & 183.87 & 222.02 \\
\hline UB & 406.00 & 1404.00 & 78.53 & 172.95 & 22.74 & 42.28 & 81.60 & 142.72 & 134.28 & 232.31 \\
\hline Wait & min_A & max_A & min_P & max_P & min_ED & max_ED & min_Cl & max_Cl & min_C2 & max_C2 \\
\hline AYG & 2224.00 & 2686.00 & 213.64 & 272.94 & 53.21 & 58.48 & 79.15 & 153.66 & 111.42 & 201.73 \\
\hline FS & 2654.00 & 2705.00 & 273.44 & 279.78 & 58.13 & 58.69 & 109.84 & 110.79 & 254.97 & 261.61 \\
\hline SS & 1532.00 & 1559.00 & 195.30 & 198.71 & 44.17 & 44.55 & 127.33 & 128.76 & 262.11 & 267.27 \\
\hline UB & 1532.00 & 2705.00 & 195.30 & 279.78 & 44.17 & 58.69 & 79.15 & 153.66 & 111.42 & 267.27 \\
\hline Fail & min_A & max_A & min_P & max_P & min_ED & max_ED & min_Cl & max_Cl & min_C2 & $\max \_C 2$ \\
\hline AYG & 1168.00 & 1576.00 & 159.44 & 194.99 & 38.56 & 44.80 & 107.23 & 116.29 & 94.87 & 222.87 \\
\hline FS & 872.00 & 1083.00 & 150.23 & 167.78 & 33.32 & 37.13 & 111.29 & 131.96 & 126.77 & 169.93 \\
\hline SS & 1037.00 & 1083.00 & 145.98 & 157.15 & 36.34 & 37.13 & 146.73 & 149.17 & 258.53 & 270.05 \\
\hline UB & 872.00 & 1576.00 & 145.98 & 194.99 & 33.32 & 44.80 & 107.23 & 149.17 & 94.87 & 270.05 \\
\hline
\end{tabular}




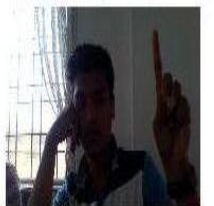

(a)

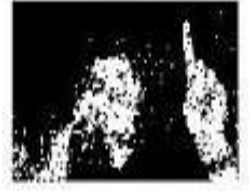

(d)

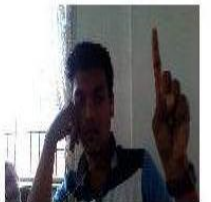

(b)

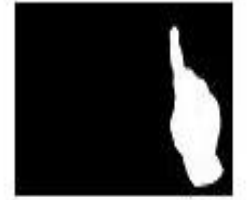

(e)

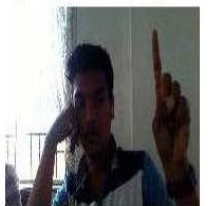

(c)

Figure. 8 Pre-processing steps incorporated in real time hand segmentation using adaptive skin colour modelling:

(a) The original input image, (b) RGB compensated, (c)

Light compensated, (d) Skin segmented, (e) Binary

filtered, and (f) ROI cropped image respectively

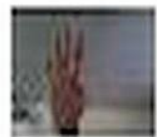

(a)

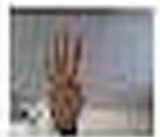

(b)

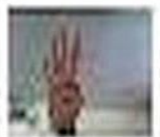

(c)

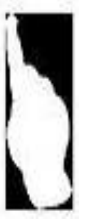

(f)

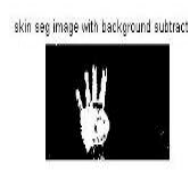

Coppes mas:

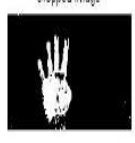

Figure. 9 Recognizing ISL number four using adaptive skin colour modelling: (a) Original input image, (b) RGB compensated, and (c) light compensated

Table 7. Confusion matrix of signer1, performing gestures in front of the camera

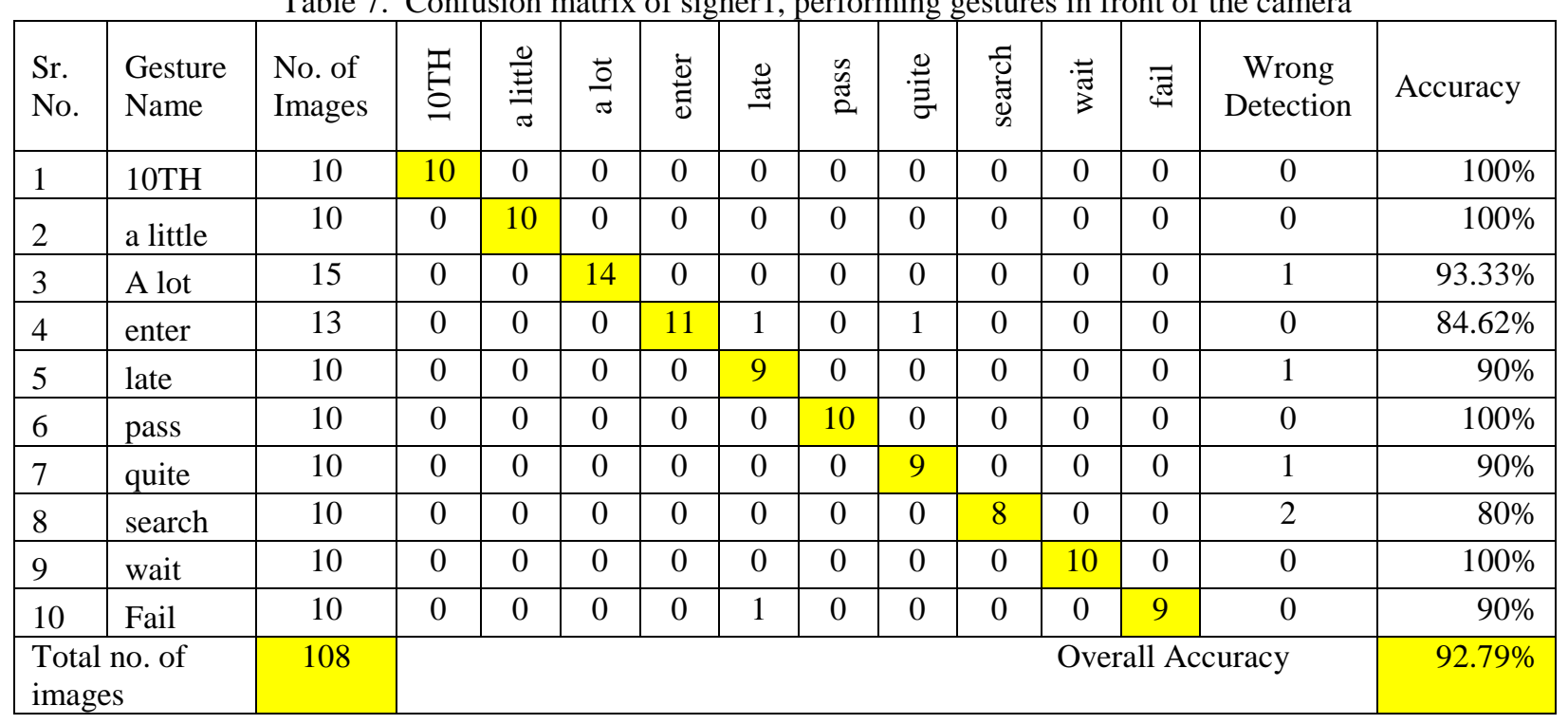

Table 8. Confusion matrix of signer 2, performing gestures in front of the camera

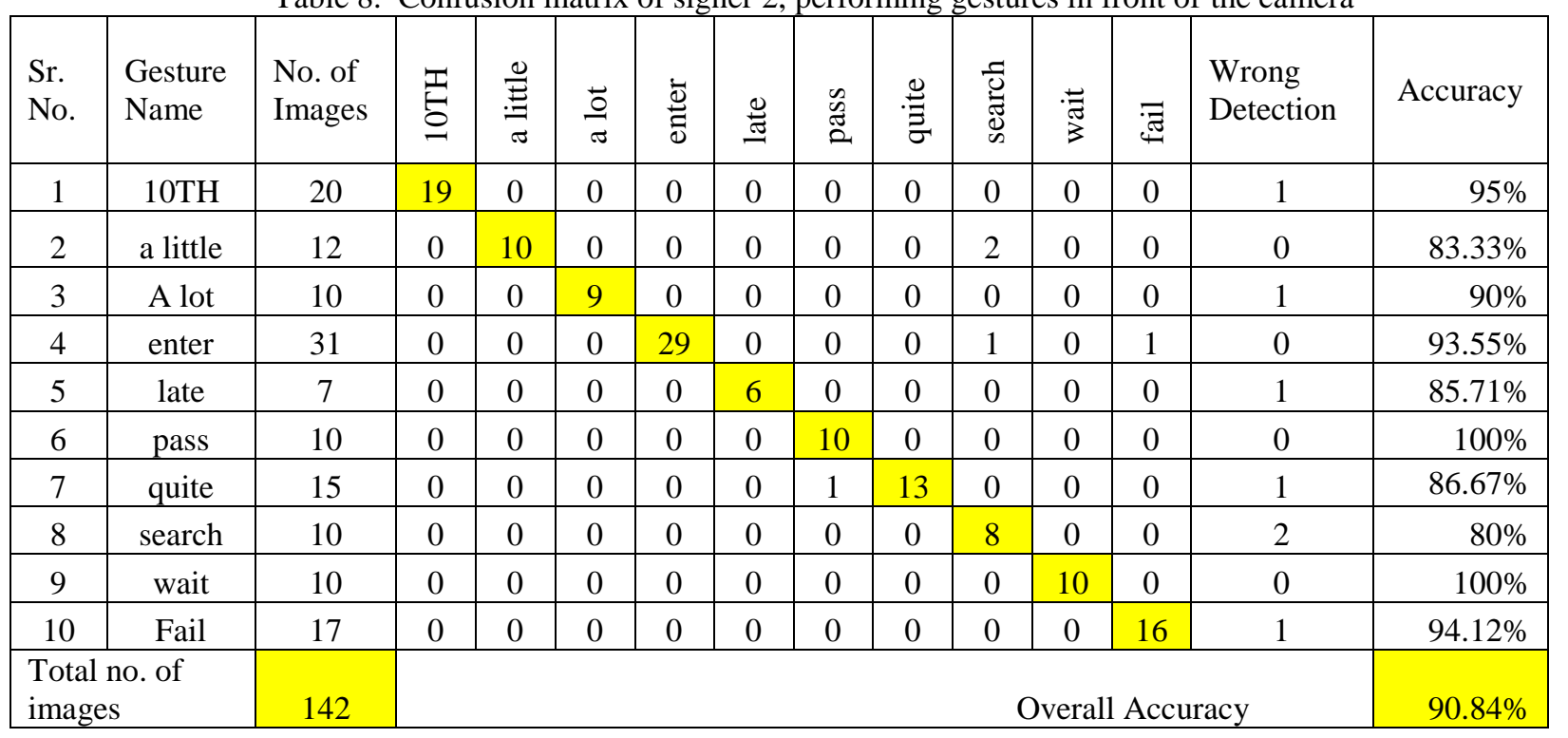


Table 9. Confusion matrix for Aliyawar Jung video dataset

\begin{tabular}{|c|c|c|c|c|c|c|c|c|c|c|c|c|c|c|}
\hline $\begin{array}{l}\text { Sr. } \\
\text { No. }\end{array}$ & $\begin{array}{l}\text { Gesture } \\
\text { Name }\end{array}$ & $\begin{array}{l}\text { No. of } \\
\text { Images }\end{array}$ & 声 & $\underset{\sigma}{\stackrel{\Xi}{\Xi}}$ & $\frac{\pi}{\sigma}$ & $\stackrel{\bar{\Xi}}{\stackrel{ \pm}{0}}$ & 䒕 & $\begin{array}{l}\tilde{E} \\
\tilde{\Xi} \\
\tilde{a}\end{array}$ & $\cdot \stackrel{\mathscr{\Xi}}{\Xi}$ & 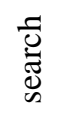 & : & : & $\begin{array}{l}\text { Wrong } \\
\text { Detection }\end{array}$ & Accuracy \\
\hline 1 & $10 \mathrm{TH}$ & 11 & 11 & 0 & 0 & 0 & 0 & 0 & 0 & 0 & 0 & 0 & 0 & $100.00 \%$ \\
\hline 2 & a little & 10 & 0 & 9 & 0 & 0 & 0 & 0 & 0 & 0 & 0 & 0 & 1 & $90 \%$ \\
\hline 3 & a lot & 7 & 0 & 0 & 7 & 0 & 0 & 0 & 0 & 0 & 0 & 0 & 0 & $100.00 \%$ \\
\hline 4 & enter & 11 & 0 & 0 & 0 & 10 & 1 & 0 & 0 & 0 & 0 & 0 & 0 & $90.91 \%$ \\
\hline 5 & late & 27 & 0 & 0 & 0 & 0 & 23 & 0 & 0 & 0 & 1 & 0 & 3 & $85.19 \%$ \\
\hline 6 & pass & 18 & 0 & 0 & 0 & 0 & 0 & 16 & 0 & 0 & 0 & 0 & 2 & $88.89 \%$ \\
\hline 7 & quite & 29 & 0 & 0 & 0 & 0 & 0 & 1 & 27 & 0 & 0 & 1 & 0 & $93.10 \%$ \\
\hline 8 & search & 21 & 0 & 0 & 0 & 0 & 0 & 0 & 0 & 18 & 0 & 0 & 3 & $85.71 \%$ \\
\hline 9 & wait & 9 & 0 & 0 & 0 & 0 & 0 & 0 & 0 & 0 & 9 & 0 & 0 & $100 \%$ \\
\hline 10 & fail & 10 & 0 & 0 & 0 & 0 & 0 & 0 & 0 & 0 & 0 & 9 & 1 & $90 \%$ \\
\hline $\begin{array}{l}\text { Total } \\
\text { imag }\end{array}$ & & 153 & & & & & & & & & \multicolumn{3}{|c|}{ Overall Accuracy } & $92.64 \%$ \\
\hline
\end{tabular}

\section{Conclusion}

A real-time skin colour segmentation is a prime concern in posture/hand gesture based sign language recognition. The proposed ASCM method separate posture/hand gesture without any marker on any background by taking into account foreground skin colour. The proposed work is segmenting dynamic posture/hand gesture done in front of the camera or from images or video database. The values of $\mathrm{YCgCr}$ determined at runtime. The $\mathrm{YCgCr}$ bound values are used to segment foreground skin colour from skin / non-skin colour background. The algorithm deployed on the open source Raspberry-Pi embedded image processing hardware platform adapt any skin tone /colour and segment face/ hand gestures of the signer. The overall accuracy including ISL standard database is $92.09 \%$. In future, the recognition accuracy and time would be improved by incorporating additional features and utilising all 4 GPUs of R-Pi.

\section{References}

[1] P. Kakumanu, S. Makrogiannis, and N. Bourbakis, "A survey of skin-colour modelling and detection methods", Pattern Recognit., Vol. 40, No. 3, pp. 1106-1122, 2007.

[2] G. Yang, H. Li, L. Zhang, and Y. Cao, "Research on a Skin Color Detection Algorithm Based on Self-adaptive Skin Color Model", In: Proc. of the 2010 Int. Conf. Commun. Intell. Inf. Secur., pp. 266-270, 2010.

[3] Y. Luo and Y.-P. Guan, "Adaptive skin detection using face location and facial structure estimation", IET Comput. Vis., Vol. 11, No. 7, pp. 550-559, 2017.
[4] Z. H. Al-Tairi, R. W. Rahmat, M. Iqbal Saripan, and P. S. Sulaiman, "Skin segmentation using YUV and RGB colour spaces", J. Inf. Process. Syst., Vol. 10, No. 2, pp. 283-299, 2014.

[5] P. H. Chen, Y. C. Yang, and L. M. Chang, "Automated bridge coating defect recognition using adaptive ellipse approach", Autom. Constr., Vol. 18, No. 5, pp. 632-643, 2009.

[6] J. Singha and K. Das, "Indian Sign Language Recognition Using Eigen Value Weighted Euclidean Distance-Based Classification Technique", arXiv Prepr. arXiv1303.0634, Vol. 4, No. 2, pp. 188-195, 2013.

[7] S. L. Phung, A. Bouzerdoum, and D. Chai, "Skin segmentation using colour pixel classification: analysis and comparison", IEEE Trans. Pattern Anal. Mach. Intell., Vol. 27, No. 1, pp. 148-154, 2005.

[8] J. Yong, A. Zhauip, and T. Tan, "Adaptive skin detection using multiple cues", In: Proc. of the International Conference on Image Processing, pp. 901-904, 2004.

[9] N. Vandenbroucke, L. Macaire, and J.-G. Postaire, "Color pixels classification in a hybrid colour space", In: Proc. 1998 Int. Conf. Image Process, pp. 176-180, 1998.

[10] W. Chen, K. Wang, H. Jiang, and M. Li, "Skin colour modelling for face detection and segmentation: a review and a new approach", Multimedia. Tools Appl., pp. 839-862, 2014.

[11] P. Dondi, M. Porta, and L. Lombardi, "Development of gesture-based humancomputer interaction applications by fusion of depth and colour video streams", IET Comput. Vis., Vol. 8, No. 6, pp. 568-578, 2014.

[12] E. Stergiopoulou, K. Sgouropoulos, N. Nikolaou, 
N. Papamarkos, and N. Mitianoudis, "Real-time hand detection in a complex background", Eng. Appl. Artif. Intell., Vol. 35, pp. 54-70, 2014.

[13] A. Y. Dawod, J. Abdullah, and M. J. Alam, "Adaptive skin colour model for hand segmentation", In: Proc. of the ICCAIE 2010 2010 Int. Conf. Comput. Appl. Ind. Electron., No. Iccaie, pp. 486-489, 2010.

[14] M. Shoyaib, M. Abdullah-Al-Wadud, and O. Chae, "A skin detection approach based on the Dempster-Shafer theory of evidence", Int. J. Approx. Reason., Vol. 53, No. 4, pp. 636-659, 2012.

[15] H. Hikawa and K. Kaida, "Novel FPGA implementation of hand sign recognition system with SOM-Hebb classifier", IEEE Trans. Circuits Syst. Video Technol., Vol. 25, No. 1, pp. 153-166, 2015.

[16] X. Zhang, X. Chen, Y. Li, V. Lantz, K. Wang, and J. Yang, "A framework for hand gesture recognition based on accelerometer and EMG sensors", IEEE Trans. Syst. Man, Cybern. Part ASystems Humans, Vol. 41, No. 6, pp. 10641076, 2011.

[17] M. Zhao, P. Li, and L. Wang, "A novel complete face detection method in colour images", In: Proc. of Image Signal Process, 2010 3rd Int. Congr., Vol. 4, pp. 1763-1766, 2010.

[18] R. Kaluri and P. Ch, "Sign Gesture Recognition Using Modified Region Growing Algorithm and Adaptive Genetic Fuzzy Classifier", International Journal of Intelligent Engineering and Systems, Vol. 9, No. 4, pp. 225-233, 2016.

[19] R. Kaluri and P. CH, "An Enhanced Framework for Sign Gesture Recognition using Hidden Markov Model and Adaptive Histogram Technique", International Journal of Intelligent Engineering and Systems, Vol. 10, No. 3, pp. 11-19, 2017. 